\title{
Taxonomic-faunistic notes on the Philodromidae (Aranei) of Turkey
}

\section{Таксономически-фаунистические заметки о пауках семейства Philodromidae Турџии}

\author{
D.V. Logunov ${ }^{1} \&$ K.B. Kunt ${ }^{2}$ \\ A.B. Аогунов ${ }^{1}$, К.Б. Кунт ${ }^{2}$
}

${ }^{1}$ The Manchester Museum, University of Manchester, Oxford Road, Manchester M13 9PL UK. E-mail: dmitri.v.logunov@manchester.ac.uk
(corresponding author).
Манчестерский Музей, Университет Манчестера, Оксфорд Роуд, Манчестер, M13 9PL Великобритания.
${ }^{2}$ Museum of the Turkish Arachnological Society, Eserkoy Sitesi, 9/A Blok, No:7, TR-06530 Ümitköy, Ankara Turkey. E-mail:
chaetopelma@gmail.com
Музей Турецкого Арахнологического Общества, Анкара Турция.

KEY WORDS: Philodromidae, Turkey, new records, new species.

КЛЮЧЕВЫЕ СЛОВА: Philodromidae, Турция, новые находки, новые виды.

ABSTRACT. This paper presents taxonomic-faunistic notes on 18 species of Philodromidae of Turkey. Artanes is re-elevated to generic rank, with 17 valid species to be included in it. Nine new combinations are proposed (all ex Philodromus): A. femurostriatus (Muster, 2009) comb.n., A. johani (Muster, 2009) comb.n., A. laricium (Simon, 1875) comb.n., A. maghrebi (Muster, 2009) comb.n., A. parietalis (Simon, 1875) comb.n., A. penteri (Muster, 2009) comb.n., A. pinetorum (Muster, 2009) comb.n., A. utotchkini (Marurik, 1991) comb.n. and A. validus (Gertsch, 1933) comb.n. Two species, Artanes bucaensis sp.n. ( + , from İzmir Province) and Thanatus nitidus sp.n. ( + , from Adryaman and Gaziantep Provinces), are described as new. Four species, viz. Philodromus bistigma Simon, 1870, $P$. pulchellus Lucas, 1846, Thanatus fabricii (Audouin, 1827) and Thanatus sabulosus (Menge, 1875), are recorded new to the Turkish fauna. In total, the philodromid fauna of Turkey is currently known to contain 37 species.

РЕЗЮМЕ. Данная работа представляет собой таксономически-фаунистические заметки по 18 видам Philodromidae Турции. Восстановлен родовой ранг Artanes, в который входит 17 валидных видов. Предложено 9 новых комбинаций (все из Philodromus): A. femurostriatus (Muster, 2009) comb.n., A. johani (Muster, 2009) comb.n., A. laricium (Simon, 1875) comb.n., A. maghrebi (Muster, 2009) comb.n., A. parietalis (Simon, 1875) comb.n., A. penteri (Muster, 2009) comb.n., A. pinetorum (Muster, 2009) comb.n., A. utotchkini (Marurik, 1991) comb.n. и A. validus (Gertsch, 1933) comb.n. Два вида, Artanes bucaensis sp.n. ( sp.n. (虫, из пров. Адияман и Гаинзатеп), описаны как новые. Четыре вида, а именно Philodromus bistigma Simon, 1870, P. pulchellus Lucas, 1846,
Thanatus fabricii (Audouin, 1827) и Thanatus sabulosus (Menge, 1875), впервые указываются для фауны Турции. Всего, фауна филодродмид Турции в настоящее время насчитывает 37 видов.

\section{Introduction}

According to the latest checklist by Demir [2008], the Philodromidae fauna of Turkey numbers 27 species, of which ten species are known only from a single locality. Records of some species remain doubtful and require verification based upon reference to the pertinent material. For instance, Philodromus lividus Simon, 1875 was recoded by Kulszyński [1903] from Bursa, but its confirmed distribution is restricted to the western Mediterranean, from Spain and Algeria eastward as far as Italy [see Muster \& Thaler, 2004]. Another example is the record of Thanatus lineatipes Simon, 1870 from İzmir [see Pavesi, 1876]. This species remains known from a few records by Simon [e.g., 1932] and an obscure record from Georgia [Mcheidze, 1997]; furthermore, its taxonomic status requires revision. It is highly unlikely that this species occurs in Turkey or in neighbouring Georgia. After the publication of Demir's [2008] checklist, four additional philodromid species have been recorded or described from Turkey by Logunov \& Huseynov [2008] and Muster [2009a]. Thus, to date, 31 species of three genera of Philodromidae have been reported from Turkey. The main purpose of this work is to present an annotated checklist of original, newly collected material on the Turkish philodromids, and to clarify and to map the distribution of studied species within Turkey. A total of 18 species of four genera is surveyed below in alphabetic order, with two species being described as new and four being newly reported for the Turkish spider fauna. 
Specimens for this study were borrowed from and/ or deposited in the following museum and institute collections: LMNM= National Museums Liverpool, Liverpool, UK (Mr. G. Knight); MTAS = Museum of Turkish Arachnological Society, Ankara, Turkey (Mr K.B. Kunt); ZMUM= Zoological Museum of the Moscow State University, Moscow, Russia (Dr. K.G. Mikhailov).

The terminology of male and female genital morphology mostly follows Logunov [1996] and Muster [2009a]. Abbrevations used in the text: a.s.1. - above see level, D — described, Distr. — district, nr. - near, Mt. - mountain, Prov. - province, Vil. - Village. Abbrevations used in the measurement data: AME anterior median eye, ALE - anterior lateral eye, AMEAME - distance between AMEs, AME-ALE - distance between AME and ALE, DTA - dorsal tibial apophysis, Fm - femur, MOA - median ocular area, MOA-WA - anterior width of MOA, MOA-WP posterior width of MOA, MOA-L - length of MOA, Mt — metatarsus, PME — posterior median eye, PLE posterior lateral eye, PME-PME distance between PMEs, PME-PLE - distance between PME and PLE, $\mathrm{Pt}$ - patella, RTA - retrolateral tibial apophysis, Tb tibia. The sequence of leg segments in measurement data is as follows: femur + patella + tibia + metatarsus + tarsus (total). All measurements are in mm. Abbreviations used in defining the position of leg spines: ap apical, d - dorsal, pr - prolateral, $\mathrm{rt}$ - retrolateral, $\mathrm{v}$ ventral. For the leg spination the system adopted is that used by Ono [1988]. Collectors' names are abbreviated as follows: CF - C. Felton, SJ - S. Judd; KBK K.B. Kunt; YMM - Yu.M. Marusik; EAY — E.A. Yağmur.

Only the most reliable sources for identification of the studied species are given under 'Identification'; for a complete set of taxonomic references see Platnick [2010]. All the earlier regional taxonomic references and/or faunistic records were provided by Demir [2008], in his checklist of the Philodromidae of Turkey, and in three subsequent works by Logunov \& Huseynov [2008] and Muster [2009a].

\section{Survey of species}

\section{Artanes Thorell, 1870}

Type species: Araneus margaritatus Clerck, 1757, by subsequent designation by Thorell [1870].

The neglected genus Artanes is a well-defined taxon within the Philodromidae [see Koch, 1837: sub Artamus; Thorell, 1870; Gertsch, 1933: sub Hordromoides; etc.]. The most recent phylogenetic analysis of the family [see Muster, 2009a] revealed it to be a distinct lineage strongly supported by a number of autapomorphies such as the leg formula (legs III longer than legs IV), the presence of three tibial apophyses, of which DTA is longest, and kidney-shaped receptacula. Yet, further reliable diagnostic characters are as follows: the flat, pentagonal carapace; Tb I with not less than four pairs of ventral spines; cymbium with dorsal bulge; spermathecae without copulatory ducts; and others [see Muster, 2009a: p. 145]. The latter author [Ibid. Cit: p. 141] came to the conclusion that the "generic rank of Artanes would be justified", but purely due to a formal reason (viz., that this decision "would render a great proportion of Philodromus paraphyletic", as if this genus with almost 250 described species was not paraphyletic already) this taxonomic act was not accepted, leaving Artanes as a subgenus of Philodromus (s.lat.).

In our opinion, a re-elevation of Artanes to the genus of its own, as argued earlier by Koch [1837: sub Artamus], Thorell [1870] and recently by Muster [2009a], is highly justified and is therefore accepted here. Currently the genus consists of 17 valid species (see Table 1), one of which is described here as new. The genus Artanes is likely to be more diverse. All the known species inhabit vertical surfaces (tree trunks or rocks) and are difficult to collect unless special methods are used. As a result, the majority of species are poorly presented in museum collections and are clearly under-recorded. The genus is almost restricted to the Palaearctic Region, with a clear centre of diversity in the Mediterranean; only one species of Artanes has been reported from eastern United States (see Table 1).

\section{Artanes bucaensis sp.n.}

Figs 1-3; Map 1.

TYPE. The $\sigma^{7}$ holotype (MTAS) from Turkey, Kaynaklar Vil. [38²1'39.5"N, 27¹7'17.9"E], Buca Distr., İzmir Prov., 04.04.2009, KBK.

ETYMOLOGY. From the locality, Buca District (İzmir Province of Turkey), from where the holotype was collected.

DIAGNOSIS. By the conformation of its tibial apophyses, this new species is most similar to A. blanckei from the central part of the Tyrrhenian Islands and southern Italy [cf. Muster, 2009a: figs 18a-c], but can be reliably distinguished from it by the wider and much stronger RTA (Figs 1,3) and the shorter DTA which is also of different shape (Fig. 2). Besides, A. bucaensis sp.n. has a well-developed conductor process (arrowed in Fig. 1); poorly developed and not visible in A. blanckei.

DISTRIBUTION. The type locality only (Map 1).

DESCRIPTION. MALE (the holotype). Measurements. Carapace 1.90 long, 2.15 wide. Ocular area: MOA-WA 0.33, MOA-WP 0.44, MOA-L 0.47. Eyes and interdistances: AME 0.09, ALE 0.15, PME 0.09, PLE 0.14, AME-AME 0.14, AME-ALE 0.06, PMEPME 0.21, PME-PLE 0.14. Abdomen 2.25 long, 2.00 wide. Chelicera length 0.68 . Clypeus height 0.28 . Length of leg segments: I $2.55+1.10+2.25+2.12+1.20$ (9.22); leg II $3.38+1.25+2.88+2.65+1.43(11.59)$; III $2.80+1.10+2.68+2.28+1.15(10.01)$; IV $2.58+$ $0.88+2.13+2.10+1.08$ (8.77). Leg spination of leg I: Fm d 1-1-1, pr 1-1-0; Tb d 1-0-1, pr and rt 0-1-0, v 12-2-2-2; Mt pr 0-1-1ap, rt 1-1-1ap, v 2-2-2. Colouration. Carapace: brownish in its rear half and whitish 
Table 1. Valid species of Artanes Thorell, 1870 and their distribution. Таблица 1. Валидные виды рода Artanes Thorell, 1870 и их распространение.

\begin{tabular}{|c|c|c|}
\hline Species & Distribution & References* \\
\hline A. blanckei Wunderlich, $1995\left(\mathrm{O}^{7}+\right)$ & $\begin{array}{l}\text { Sardinia, Corsica and } \\
\text { continental Italy }\end{array}$ & Muster [2009a: figs 3, 18, 23] \\
\hline A. bucaensis sp.n. $\left(\mathrm{O}^{7}\right)$ & Turkey (TL) & Figs $1-3$ \\
\hline A. calidus (Lucas, 1846) $\left(\mathrm{O}^{7}+\right)$ & Northern Africa & Muster [2009a: figs 16,28$]$ \\
\hline A. femurostriatus (Muster, 2009) comb.n. $\left(\mathrm{O}^{\top}+\right)$ & Eastern Mediterranean & Muster [2009a: figs 12,29$]$ \\
\hline A. fuscomarginatus (De Geer, 1778) $\left(\sigma^{\top}+\right)$ & European temperate range & $\begin{array}{l}\text { Almquist [2006: fig. 395], Muster } \\
\text { [2009a: figs 5, 11, 22] }\end{array}$ \\
\hline A. johani (Muster, 2009) comb.n. $\left(\mathrm{O}^{7}+\right)$ & Crete (TL) & Muster [2009a: figs 17, 27] \\
\hline A. laricium (Simon, 1875) comb.n. $\left(\mathrm{O}^{7}+\right)$ & Central Europe (the Alps) & Muster [2009a: figs 13, 26] \\
\hline A. maghrebi (Muster, 2009) comb.n. ( ( + ) & Northern Algeria & Muster [2009a: fig. 21] \\
\hline A. margaritatus (Clerck, 1757) $\left(\mathrm{O}^{7}+\right)$ & $\begin{array}{l}\text { Trans-Palaearctic temperate } \\
\text { range }\end{array}$ & $\begin{array}{l}\text { Almquist [2006: fig. 396], Muster } \\
\text { [2009a: figs } 4,10,20]\end{array}$ \\
\hline A. marusiki Logunov, $1997\left(0^{7}+\right)$ & Mountains of South Siberia & Logunov [1997: figs 1-6] \\
\hline A. parietalis (Simon, 1875) comb.n. $\left(\mathrm{O}^{7}+\right)$ & Pyrenean Peninsula & Muster [2009a: figs 19, 31] \\
\hline A. penteri (Muster, 2009) comb.n. ( $(+)$ & $\begin{array}{l}\text { The Balkans and Eastern } \\
\text { Caucasus (Lenkoran) }\end{array}$ & Muster [2009a: fig. 30] \\
\hline A. pinetorum (Muster, 2009) comb.n. $\left(\mathrm{O}^{7}+\right.$ ) & The Mediterranean & Muster [2009a: figs 2, 9, 15, 25] \\
\hline A. poecilus Thorell, $1872\left(\sigma^{\top}+\right)$ & $\begin{array}{l}\text { European-Central Asian } \\
\text { range }\end{array}$ & $\begin{array}{l}\text { Almquist [2006: fig. 397], Muster } \\
\text { [2009a: figs 6, 8, 14, 24] }\end{array}$ \\
\hline A. spinitarsis (Simon, 1895) $\left(\mathrm{O}^{7}+\right)$ & $\begin{array}{l}\text { Far East (Russia, Korea, NE } \\
\text { China, Japan) }\end{array}$ & $\begin{array}{l}\text { Ono \& Ban [2009: } 480, \text { figs } 62- \\
63]\end{array}$ \\
\hline A. utotchkini (Marurik, 1991) comb.n. $\left(\mathrm{O}^{7}+\right)$ & North-Eastern Siberia & Marusik [1991: figs 3.4-6, 4.8-9] \\
\hline 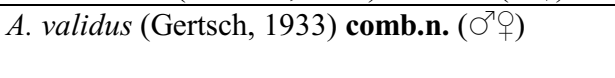 & Eastern United States & $\begin{array}{l}\text { Dondale \& Redner [1975: figs } 1- \\
4,6]\end{array}$ \\
\hline
\end{tabular}

* Only most recent/important references are given, for a complete set of taxonomic references see Platnick [2010]. Abbreviation (TL) means that the species is known from the type locality only.

yellow in front half, including ocular area and clypeus; and with brown margins. Sternum, maxillae and labium yellow, tinged with brown. Chelicerae yellowish brownish. Abdomen: dorsum whitish, with X-shaped dark brown figure; venter brownish yellow. Book-lung covers whitish yellow. Spinnerets brown. All legs whitish yellow, with dark brown patches, but femora I dark brown vertrally. Palps whitish yellow, with ventral side of femora dark brown and brown tegulum. Palpal structure as in Figs $1-3$.

FEMALE unknown.

Philodromus Walckenaer, 1826

Philodromus (s.lat.) is a large genus, with over 240 species described worldwide [Platnick, 2010]. The Turkish fauna consists of 22 recorded species [Demir, 2008; Logunov \& Huseynov, 2008; Muster, 2009a; present data].

Philodromus bistigma Simon, 1870 Map 1. , 69].

Identification: Muster et al. [2007: 51, figs 6, 10, 24, 35, 46,

MATERIAL. Turkey: $10^{7} 4$ क (ZMUM), Dilek Peninsula National Park $\left[37^{\circ} 39.333^{\prime} \mathrm{N}, 27^{\circ} 02.882^{\prime} \mathrm{E}\right]$, Güzelçamlı Vil., Kuşadası Distr., Aydın Prov., 306 m a.s.1., 7.06.2009, YMM.

DISTRIBUTION. A Mediterranean species known to date from Spain and northern Algeria in the west to
Israel in the east [Muster et al., 2007: fig. 83]. The record from Turkey lies in north-easternmost limit of the species known range. This is a new record to the Turkish spider fauna.

Philodromus cespitum (Walckenaer, 1802) Map 1

Identification: Kubcová [2004: 293, fig. 12]; Muster \& Thaler [2004: 313, figs 12, 22, 32, 33].

MATERIAL. TURKey: $1 \mathrm{O}^{\top}$ (MTAS), Pınarbaș1 [38¹0'56.89"N, 37¹3'12.93"E], Elbistan Distr., Kahramanmaraş Prov., 31.05.2008, KBK; $10^{7}$ (LMNM), Seyhkoyun Vil. [40 $58^{\prime} 24^{\prime \prime N}, 35^{\circ} 34^{\prime} 55^{\prime \prime} \mathrm{E}$ ], c. $10 \mathrm{~km} \mathrm{~W}$ of Havza, Samsun Distr., young mixed deciduous woodland with disturbed edge, 16.05.1994, SJ \& CF; 1 \& (LMNM), Kovada Gölü National Park [37³8'56.54"N, 3052'4.51"E], Isparta Prov., sparsely vegetated lakeside shingle, 20.06.1993, CF.

DISTRIBUTION. This seems to be a widespread, circum-Holarctic species [Muster \& Thaler, 2004], but records from eastern Siberia may belong to a separate species [see Marusik et al., 2000: p. 89].

Philodromus collinus C.L. Koch, 1835 Map 1

Identification: Kubcová [2004: 293, fig. 9]; Muster \& Thaler [2004: 315, figs 9, 23, 31].

MATERIAL. TURKEY: $10^{7}$ (ZMUM), Erikli Vil. [40²4.302'N, $\left.42^{\circ} 17.809^{\prime} E\right]$, Meydancık Town, Șavşat Distr., Artvin Prov., 1141 m a.s.l., 12.06.2009, YMM.

DISTRIBUTION. A European species known from Algeria in the west throughout the Mediterranean to 


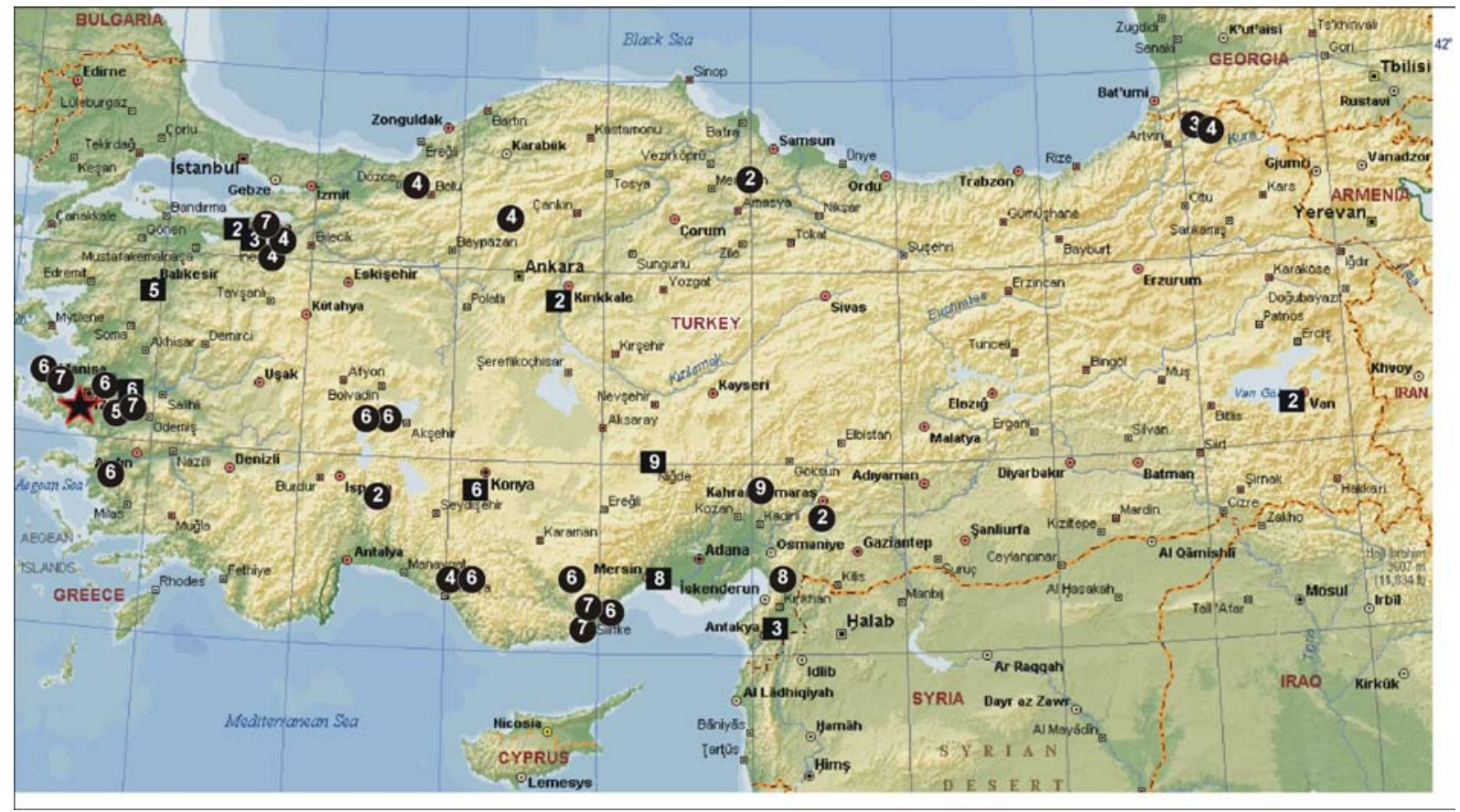

Map 1. Collection localities of Philodromidae in Turkey: Artanes bucaensis ( $\star$ ), Philodromus bistigma (1), P. cespitum (2), P. collinus (3), P. dispar (4), P. longipalpis (5), P. lunatus (6), P. pulchellus (7), P. rufus (8) and Thanatus atratus (9). Circles - original data, squares - literature-derived data [Muster \& Thaler, 2004; Demir et al., 2008; Demir, 2008].

Карта 1. Находки Philodromidae в Турции: Artanes bucaensis ( $\star$ ), Philodromus bistigma (1), P. cespitum (2), P. collinus (3), P. dispar (4), P. longipalpis (5), P. lunatus (6), P. pulchellus (7), P. rufus (8) and Thanatus atratus (9). Кружки - оригинальные данные, квадраты - литературные данные [Muster \& Thaler, 2004; Demir et al., 2008; Demir, 2008].

Turkey in the east [Muster \& Thaler, 2004; present data], northwards to the European part of Russia and Scandinavia [Mikhailov, 1997; Almquist, 2006].

\section{Philodromus dispar Walckenaer, 1826 Map 1}

Identification: Roberts [1995: 170, plate 10]; Almquist [2006 459, figs 393a-f].

MATERIAL. TURKEY: $1+$ (MTAS), entrance of Oylat Cave [39॰56'35.11"N, 29³5'27.44"E], İnegöl Distr., Bursa Prov., 03.06.2009, KBK; 2 우 (ZMUM), Nilüfer Distr. [4007.466' N, $\left.28^{\circ} 42.105^{\prime} \mathrm{E}\right]$, Bursa Prov., $570 \mathrm{~m}$ a.s.1., 2.06.2009, YMM; 1 古 (ZMUM), Erikli Vil. [40²4.302'N, 42¹7.809'E], Meydancık Town, Savşat Distr., Artvin Prov., 1141 m a.s.1., 12.06.2009, YMM; $10^{2}$ (ZMUM), Taşatan Plateau [36 $\left.40.244^{\prime} \mathrm{N}, 32^{\circ} 10.210^{\prime} \mathrm{E}\right]$, Alanya Distr., Antalya Prov., 1208 m a.s.1., 9.06.2009, YMM; 1 (ZMUM), Soğuksu National Park [40²7'21.54"N, 32³5'36.12"E], Göllü Area, Kizilcahamam Distr., Ankara Prov., 27.05.2009, KBK; 1 व (ZMUM), Abant Locality [4040'39.36"N, 31²8'18.78"E], Bolu Prov., 28.05.2009, KBK

DISTRIBUTION. $P$. dispar is a common European temperate species [Canard, 2005], known eastwards as far as Azerbaijan [Logunov \& Huseynov, 2008]. The species was also introduced to North America (British Columbia and Washington State) [Dondale \& Redner, 1978].

Philodromus longipalpis Simon, 1870 Map 1.

Identification: Muster \& Thaler [2004: 318, figs 10, 25, 26].
MATERIAL. TURKEY: $1 \sigma^{7}$ (MTAS), Campus of University of Ege [ $\left.38^{\circ} 27^{\prime} 0.71^{\prime \prime N}, 27^{\circ} 13^{\prime} 38.75^{\prime \prime E}\right]$, Bornova Distr., İzmir Prov., 26.05.2008, KBK.

DISTRIBUTION. A Mediterranean species, known from Spain in the west to Iran and Azerbaijan in the east [Muster \& Thaler, 2004; Logunov \& Huseynov, 2008].

Philodromus lunatus Muster \& Thaler, 2004 Map 1.

Identification: Muster \& Thaler [2004: 319, figs 4, 6, 19, 26].

MATERIAL. TURKEY: $1 \sigma^{7}$ (ZMUM), c. $1 \mathrm{~km} \mathrm{~N}$ of Parlak Vil. [38 36.016'N, 26²3.254'E], Karaburun Distr., İzmir Prov., 170 m a.s.1., 6.06.2009, YMM; 2 † (ZMUM), Dilek Peninsula National

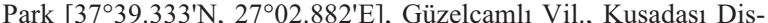
tr., Aydın Prov., 306 m a.s.1., 7.06.2009, YMM; 1 ช’ 2 of (ZMUM), Vişneli Vil. (Fetrek-2 Cave) [38²0.777'N, 27²5.271'E], Kemalpaşa

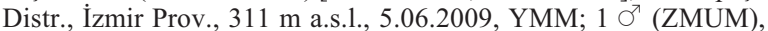
Taşatan Plateau, [36 $\left.40.521^{\prime} \mathrm{N}, 32^{\circ} 10.998^{\prime} \mathrm{E}\right]$ Alanya Distr., Antalya Prov., $1290 \mathrm{~m}$ a.s.1., 9.06.2009, YMM; $1 \sigma^{7}$ (LMNM), Sultan Mts [38 $18^{\prime} 14.24^{\prime \prime} \mathrm{N}, 31^{\circ} 27^{\prime} 22.55^{\prime \prime E}$ ], c. $10 \mathrm{~km}$ along AkşehirÇetince road, Konya Prov., mixed herbs and shrubs among young pine plantation, 19.06.1993, SJ \& CF; 1 ㅇ (LMNM), above Dað Otel [38 $20^{\prime} 55.85^{\prime \prime N}, 31^{\circ} 24^{\prime} 45.89^{\prime \prime E}$ ], Akşehir Distr., Konya Prov., mixed scrub, herbs and grasses on north-facing limestone slope, 19.09.1993, SJ \& CF; 1 क (LMNM), Narlıkuyu Town [36?26'35'N, 34?04'44'E], Silifke Distr., Mersin Prov., 29.06.1995, SJ \& CF; 1 우 (LMNM), c. $30 \mathrm{~km} \mathrm{E}$ of Mut [36 $\left.38^{\prime} 56.70^{\prime \prime} \mathrm{N}, 33^{\circ} 21^{\prime} 41.45^{\prime \prime E}\right]$, Mut Distr., Mersin Prov., 17.07.1992, CF.

DISTRIBUTION. An eastern Mediterranean species, known so far from Greece and Croatia in the west to Turkey in the east [Muster \& Thaler, 2004; present data]. 
Philodromus pulchellus Lucas, 1846 Map 1. $63,64]$.

Identification: Muster et al. [2007: 60, figs 5, 7, 21, 32, 43, 54,

MATERIAL. TURKEY: $1 \sigma^{7} 1$ (ZMUM), campus of Uludağ Univ. [40¹3.549'N, 2852.109'E], Gorukle Vil., Bursa Prov., 423 m a.s.1., 2-3.06.2009, YMM; 3 우 (ZMUM), c. $1 \mathrm{~km} \mathrm{~N}$ of Parlak Vil. [38 $36.016^{\prime} \mathrm{N}, 26^{\circ} 23.254^{\prime} \mathrm{E}$ ], Karaburun Distr., İzmir Prov., $170 \mathrm{~m}$ a.s.l., 6.06.2009, YMM; 2 우 (ZMUM), Vișneli Vil. (Fetrek-2 Cave) [ $\left.38^{\circ} 20.777^{\prime} \mathrm{N}, 27^{\circ} 25.271^{\prime} \mathrm{E}\right]$, Kemalpaşa Distr., İzmir Prov., $311 \mathrm{~m}$ a.s.1., 5.06.2009, YMM; 1 (LMNM), Silifke Distr., Sand Dunes [36 $17^{\circ} 05^{\prime \prime N}, 33^{\circ} 56^{\prime} 08^{\prime \prime E}$ ], Mersin Prov., inner dunes with damp, Salicornia dominated, halophytic vegetation and red bed, 6.05.1994, SJ \& CF; 2 OO (LMNM), c. 2 km S of Camlidere Vil. [36 52'12"N, 34 24'37"E], Mersin Prov., rocky disused quarry at side of road, dominated by Euphorbia, Mullein, Cistus and Rubus, 30.06.1995, SJ \& CF.

DISTRIBUTION. A Mediterranean species known to date from Spain and northern Algeria in the west to Egypt and Israel in the east [Muster et al., 2007: fig. 82]. The record from Turkey lies in north-easternmost limit of the known species range. This is a new record to the Turkish spider fauna.

\section{Philodromus rufus Walckenaer, 1826 Map 1.}

Identification: Segers [1989: 38, figs 1-2, 7]; Almquist [2006: 468, figs $400 \mathrm{a}-\mathrm{d}]$.

MATERIAL. TuRKEY: $1 \sigma^{\top}$ (MTAS), road over Çardak Plateau [36 50'19.10"N, 36²7'4.68"E], Hassa Distr., Hatay Prov., 25.04.2008, KBK.

DISTRIBUTION. It is a circum-Holarctic temperate species [Marusik et al., 2000].

\section{Thanatus C.L. Koch, 1837}

Thanatus is a large genus, with some 97 species described worldwide, except for Australia [Platnick, 2010]. At least 15 European and Mediterranean species remain known from single sex and/or type locality only. The Turkish fauna consists of 12 recorded species [Demir, 2008; Logunov \& Huseynov, 2008; present data], of which one is described as new.

\section{Thanatus atratus Simon, 1875$$
\text { Map } 1 .
$$

Identification: Logunov [1996: 185, figs 190-193, 198-200]; Muster \& Thaler [2003: 376, figs 3, 6, 9, 14, 15, 20-23].

MATERIAL. TURKEY: 1 (MTAS), c. $3 \mathrm{~km} \mathrm{~W}$ of Andirın [37³4'16.12"N, 36²19'32.23"E], Andırın Distr., Kahramanmaraş Prov., 02.07.2006, KBK.

DISTRIBUTION. The species is known from central Europe [Muster \& Thaler, 2003], throughout Scandinavia [Almquist, 2006] to western Siberia [Logunov, 1996]. The records from Turkey [Demir et al., 2008; present data] lie at the southernmost limits of the species distribution.

\section{Thanatus fabricii (Audouin, 1827) Map 2.}

Identification: Levy [1977: 219, figs 50-54].
MATERIAL. TURKEY: $4 O^{7} \sigma^{7}$ (ZMUM), 1 ( 9 (MTAS), Aktepe Town [36 $\left.46^{\circ} 55.55^{\prime \prime} \mathrm{N}, 36^{\circ} 29^{\prime} 58.83^{\prime \prime E}\right]$, Hassa Distr., Hatay Prov., 05.05.2008, KBK; 2 우 (MTAS), c. $1 \mathrm{~km} \mathrm{E} \mathrm{of} \mathrm{Küplüce} \mathrm{Vil.}$ [36² $45^{\prime} 19.14^{\prime \prime N}, 37^{\circ} 14^{\prime} 23.81^{\prime \prime E}$ ], Kilis Prov., 24.04.2008, KBK; 1 ( (MTAS), Aktepe Town [36 $\left.36^{\circ} 2^{\prime} 16.70^{\prime \prime} \mathrm{N}, 36^{\circ} 29^{\prime} 52.51^{\prime \prime E}\right]$, Hassa Distr., Hatay Prov., 23.04.2008, KBK; 1 (MTAS), central part of İslahiye Distr. [37'1'42.93"N, 36 37'57.95"E], Gaziantep Prov., 20.05.2008, KBK; 1 ㅇ (MTAS), Yörük Vil. [37¹5'25.56"N, 41 $\left.58^{\prime} 33.74 " E\right]$, İdil Distr., Sirnak Prov., 20.05.2009, KBK; 1 ㅇ (LMNM), sand dunes nr. Silifke [ $\left.36^{\circ} 17^{\prime} 05^{\prime \prime} \mathrm{N}, 33^{\circ} 56^{\prime} 08^{\prime \prime} \mathrm{E}\right]$, Silifke Distr., Mersin Prov., inner dunes with damp, Salicornia dominated, halophytic vegetation and red bed, 6.05.1994, SJ \& CF; $10^{7}$

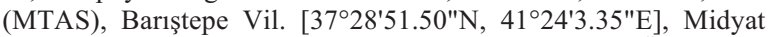
Distr., Mardin Prov., 17.05.2009, EAY; 1 क (ZMUM), Örenli Vil. [3744'47.99"N, 37³8'3.50"E], Gölbaş1 Distr., Adıyaman Prov., 09.06.2007, KBK.

DISTRIBUTION. This Mediterranean species is known to date from the Canary Islands in the west [Wunderlich, 1992], throughout Sahara, the Middle East [Levy, 1977] and the Caucasus [Logunov \& Huseynov, 2008], to Tadjikistan in the east [Lyakhov, 2000]. This is a new record to the Turkish spider fauna.

\section{Thanatus imbecillus L. Koch, 1878 Map 2.}

Identification: Lyakhov [2000: 223, figs 24-27]; Logunov \& Huseynov [2008: 124, figs 19-22].

MATERIAL. TURKEY: $2 \sigma^{\top} \sigma^{\top} 70$ (MTAS), Sarısalkım Vil. [37 $\left.5^{\prime} 24.57^{\prime \prime} \mathrm{N}, 37^{\circ} 16^{\prime} 43.19^{\prime \prime} \mathrm{E}\right]$, Gaziantep Prov., 8-12.04.2004, EAY; 6 우 (MTAS), same locality, 29.06.2004, EAY; 1 ○ (MTAS), crossroad nr. Ozanl1 [3658'37.93"N, 37¹0'18.10"E], Şahinbey Distr., Gaziantep Prov., 17.03.2007, KBK; $1 \sigma^{7}$ (MTAS), Kartalyücesi Mt. [377'45.88"N, 37¹1'23.96"E], Șehitkamil Distr., Gaziantep Prov., 28.03.2008, KBK; 3 ㅇ (MTAS), c. $2 \mathrm{~km} \mathrm{~W}$ of Fevzipaşa Town [376'5.15"N, 36³7'19.89"E], Hasanbeyli Road, İslahiye Distr., Gaziantep Prov., 24.04.2008, KBK; $2 \sigma^{7} \sigma^{7} 10$ 우 (MTAS), Guvercin Vil. [3747'25.43"N, 39³4'29.76"E], Siverek Distr., Şanlıurfa Prov., 11.04.2008, KBK; 19 (MTAS), Karahisar Vil. [37³'43.32"N, 39 $15^{\prime} 53.19^{\prime \prime}$ ], Karaca Hamlet, Tektek Mountains, Şanlıurfa Prov., 25.04.2009, KBK; 1 † (MTAS), Çalışkanlar Vil. [37 $\left.7^{\circ} 8^{\prime} 6.92^{\prime \prime N}, 38^{\circ} 42^{\prime} 46.88^{\prime \prime} E\right]$, Sanliurfa Prov., 11.04.2006, $\mathrm{KBK} ; 4$ 우 (MTAS), Yukarı Habip Vil. [37 $5^{\prime} 45.62^{\prime \prime N}$, 3754'48.54"E], Birecik Distr., Şanlıurfa Prov., 24.04.2009, KBK; 2 오 (MTAS), c. $2 \mathrm{~km} \mathrm{~S}$ of Yesilözen Vil. [37 $12^{\prime} 0.24^{\prime \prime N}$, 3757'25.10"E], Halfeti Distr., Şanlıurfa Prov., 24.04.2009, KBK;

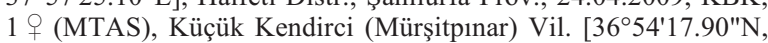
$\left.38^{\circ} 20^{\prime} 42.84^{\prime \prime E}\right]$, Suruç Distr., Sanlıurfa Prov., 12.04.2006, KBK; 1 ㅇ (MTAS), c. $1 \mathrm{~km} \mathrm{~S}$ of Argaç Vil. [37 14'16.83"N, 3753'55.98"E], Halfeti Distr., Sanlıurfa Prov., 11.05.2006, KBK; 1 (MTAS), Edebey Vil. [3650'6.88"N, 3841'8.91"E], Akçakale Distr., Şanlıurfa Prov., 22.05.2007, KBK; 1 q (MTAS), c. 2 km S

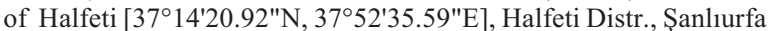
Prov., 29.03.2008, KBK; 5 ○ $^{\top} 2$ 90 (ZMUM), Çermik Distr. [38 $\left.8^{\prime} 21.03^{\prime \prime N}, 39^{\circ} 26^{\prime} 46.14^{\prime \prime E}\right]$, Diyarbakir Prov., 10.05.2007, KBK; $10^{7} 3$ 우 (ZMUM), Gerger Distr. [38²'5.86"N, 39॰4'19.46"E], Adıyaman Prov., 19.04.2008, KBK; 3 o ○ $^{\top}$ (MTAS), c. $9 \mathrm{~km} \mathrm{~W}$ of Ömerli, Hop Pass [37 $\left.22^{\prime} 28.02^{\prime \prime N}, 40^{\circ} 52^{\prime} 27.32^{\prime \prime E}\right]$, Ömerli Distr., Mardin Prov., 18.04.2007, KBK; 2 우 (MTAS),

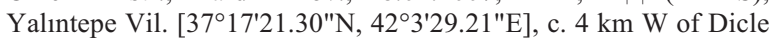
(the Tigris) River, Cizre Distr., Sirnak Prov., 12.05.2007, KBK; $1 \sigma^{7}$ (MTAS), Çardak Plateau [3650'39.06"N, 36²6'23.81"E],

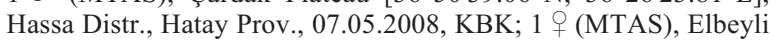
Distr. [36 $46^{\prime} 25.77^{\prime \prime N}, 37^{\circ} 28^{\prime} 33.37^{\prime \prime E}$ ], Kilis Prov., 22.04.2006, $\mathrm{KBK} ; 3$ 우 (MTAS), c. $1 \mathrm{~km} \mathrm{E}$ of Küplüce Vil. [36 $45^{\circ} 8.01$ "N, $\left.37^{\circ} 14^{\prime} 13.77^{\prime \prime E}\right]$, Kilis Prov., 24.04.2008, KBK; $1 \sigma^{7}$ (MTAS), c. 4 km SW of Yeşilli [37¹9'58.99"N, 4050'14.23"E], Yeşilli Distr., Mardin Prov., 07.04.2007, KBK; 2 + (MTAS), Kurtboğazı Dam, on the road between K1z1lcahamam and Ankara [40 21'14.09"N, 32॰40'51.39"E], Ankara Prov., 27.V.2009, KBK; 1 9 (MTAS), c. 5 $\mathrm{km}$ E of Tut [37 $\left.47^{\prime} 49.63^{\prime \prime N}, 37^{\circ} 53^{\prime} 22.35^{\prime \prime E}\right]$, Tut Distr., Adiya- 


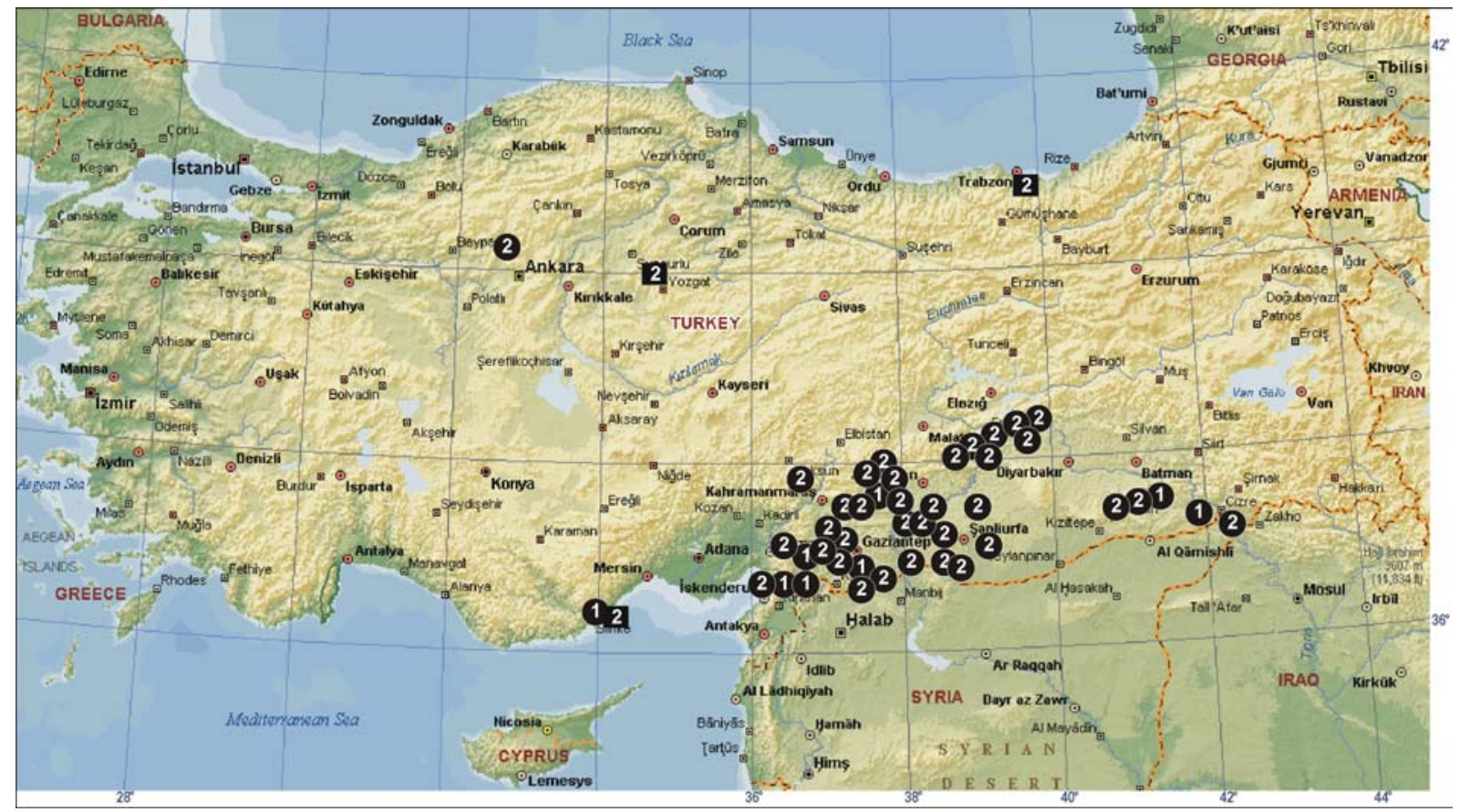

Map 2. Collection localities of Philodromidae in Turkey: Thanatus fabricii (1) and T. imbecillus (2). Circles — original data, squares literature-derived data [Logunov \& Huseynov, 2008].

Карта 2. Находки Philodromidae в Турции: Thanatus fabricii (1) и T. imbecillus (2). Кружки - оригинальные данные, квадраты литературные данные [Logunov \& Huseynov, 2008].

man Prov., 04.05.2008, KBK; 3 우 (MTAS), Eğil Distr. [38¹5'0.09"N, 404'24.99"E], Diyarbakır Prov., 13.04.2008, KBK;

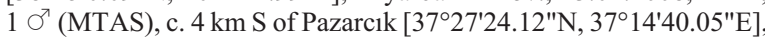
Pazarcık Distr., Kahramanmaraș Prov., 08.03.2008, KBK; 1 ơ

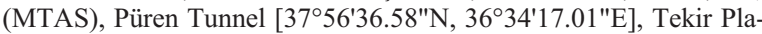
teau, Kahramanmaraş Prov., 01.05.2008, KBK; 1 \% (MTAS), c. 1 km W of Karakuyu Vil. [37²4'12.26"N, 37³8'45.57"E], Gölbas 1 Distr., Adiyaman Prov., 05.04.2007, KBK; $10^{7} 1$ क (MTAS), border of Osmaniye and Gaziantep Provinces, Kuscubeli Pass [37 $7^{\circ} 139.21^{\prime \prime N}$, $\left.36^{\circ} 33^{\prime} 31.58^{\prime \prime E}\right], 24.04 .2008$, KBK; $10^{71} 1$ ㅇ (MTAS), Petekkaya Vil. [38 $\left.5^{\prime} 32.14 " \mathrm{~N}, 39^{\circ} 26^{\prime} 20.26 " \mathrm{E}\right]$, Çermik Distr., Diyarbakır Prov., 11.04.2008, KBK; 1 ( (MTAS), Ergani Distr. [38 $18^{\circ} 50.59^{\prime \prime N}$, $\left.39^{\circ} 49^{\prime} 7.55^{\prime \prime E}\right]$, Diyarbakır Prov., 12.04.2008, KBK; $20^{7} 0^{7}$ (ZMUM), c. $5 \mathrm{~km}$ NE of Aydınlar Vil. [37039'45.08"N, 38²4'23.44"E], Gerger Distr., Adiyaman Prov., 20.04.2008, KBK; 3 우 (MTAS), Salihli Vil. [38 $\left.14^{\prime} 12.47^{\prime \prime N}, 39^{\circ} 40^{\prime} 4.43 " E\right]$, Ergani Distr., Diyarbakır Prov., 12.04.2008, KBK; 1 t (MTAS), c. 4 km E of Kaşlıca Vil.

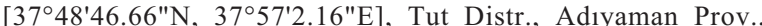
03.06.2007, KBK; 1 + (MTAS), Meryem Uşağı Vil., [37²48'6.68"N $\left.37^{\circ} 51^{\prime} 41.83^{\prime \prime E}\right]$ Tut Distr., Adiyaman Prov., 03.06.2007, KBK; $10^{7}$ (MTAS), c. $1.5 \mathrm{~km}$ E of Suvarlı Vil. [37'32'13.01"N, 37³7'21.94"E], Besni Distr., Adiyaman Prov., 06.04.2008, KBK; 1 + (MTAS), c. 2 $\mathrm{km}$ E of Küplüce Vil. [36 $45^{\circ} 8.01^{\prime \prime N}, 37^{\circ} 14^{\prime} 13.77^{\prime \prime E}$ ], Kilis Prov., 23.04.2007, KBK.

DISTRIBUTION. This is a relatively widespread species occurring from the Greek islands to Central Asia as far as Tadjikistan [Lyakhov, 2000; Logunov \& Huseynov, 2008; and the works cited therein]. The occurrence of T. imbecillus in central Europe [see Canard, 2005] needs confirmation. It is one of the commonest species of the Turkish philodromids, displaying a high variation in body size (males from one samples may twice as large as those from another) and colour (viz. the median whitish stripe of carapace can be well-marked or virtually absent).

\section{Thanatus nitidus sp.n.}

Figs 4-5; Map 4.

TYPE. The 9 holotype (MTAS) from Turkey, c. $5 \mathrm{~km}$ E of Tepecik Vil. [37048'16.18"N, $\left.38^{\circ} 1^{\prime} 38.93 " E\right]$, Tut Distr., Adıyaman Prov., 04.05.2008, KBK.

PARATYPES. TURKEY: 2 99 (ZMUM), Sarısalkım Vil. [37'5'24.57"N, 37¹6'43.19"E], Gaziantep Prov., 17.06.2004, EAY.

ETHYMOLOGY. From Latin 'nitidus' meaning 'elegant'.

DIAGNOSIS. The female of T. nitidus sp.n. is most similar to those of $T$. lesserti (Roewer, 1951) from NE Africa and the Middle East [see Levy, 1991: figs 9-10] and T. jaikensis Ponomarev, 2007 from NW Kazakhstan [see Ponomarev, 2007: fig. 10]. From the former, it can be easily distinguished by the wider, ovoid lateral guide pockets and the narrower central division (Fig. 4) and the receptacles of different shapes (Fig. 5); from the latter, it differs in having the significantly larger receptacles (Fig. 5).

DISTRIBUTION. Two localities in Turkey (Map 4).

DESCRIPTION. MALE unknown.

FEMALE (the holotype). Measurements. Carapace 2.15 long, 2.08 wide. Ocular area: MOA-WA 0.36, MOA-WP 0.48, MOA-L 0.41. Eyes and interdistances: AME 0.09, ALE 0.09, PME 0.09, PLE 0.13, AMEAME 0.20, AME-ALE 0.09, PME-PME 0.31, PMEPLE 0.33. Abdomen 2.95 long, 1.65 wide. Chelicera length 0.87 . Clypeus height 0.34 . Length of leg segments: I $2.15+0.83+1.75+1.45+1.05$ (7.23); leg II $2.55+1.03+2.05+1.73+1.15(8.51) ;$ III $2.20+0.85$ $+1.73+1.45+0.93(7.16) ;$ IV $2.65+0.90+2.13+$ 


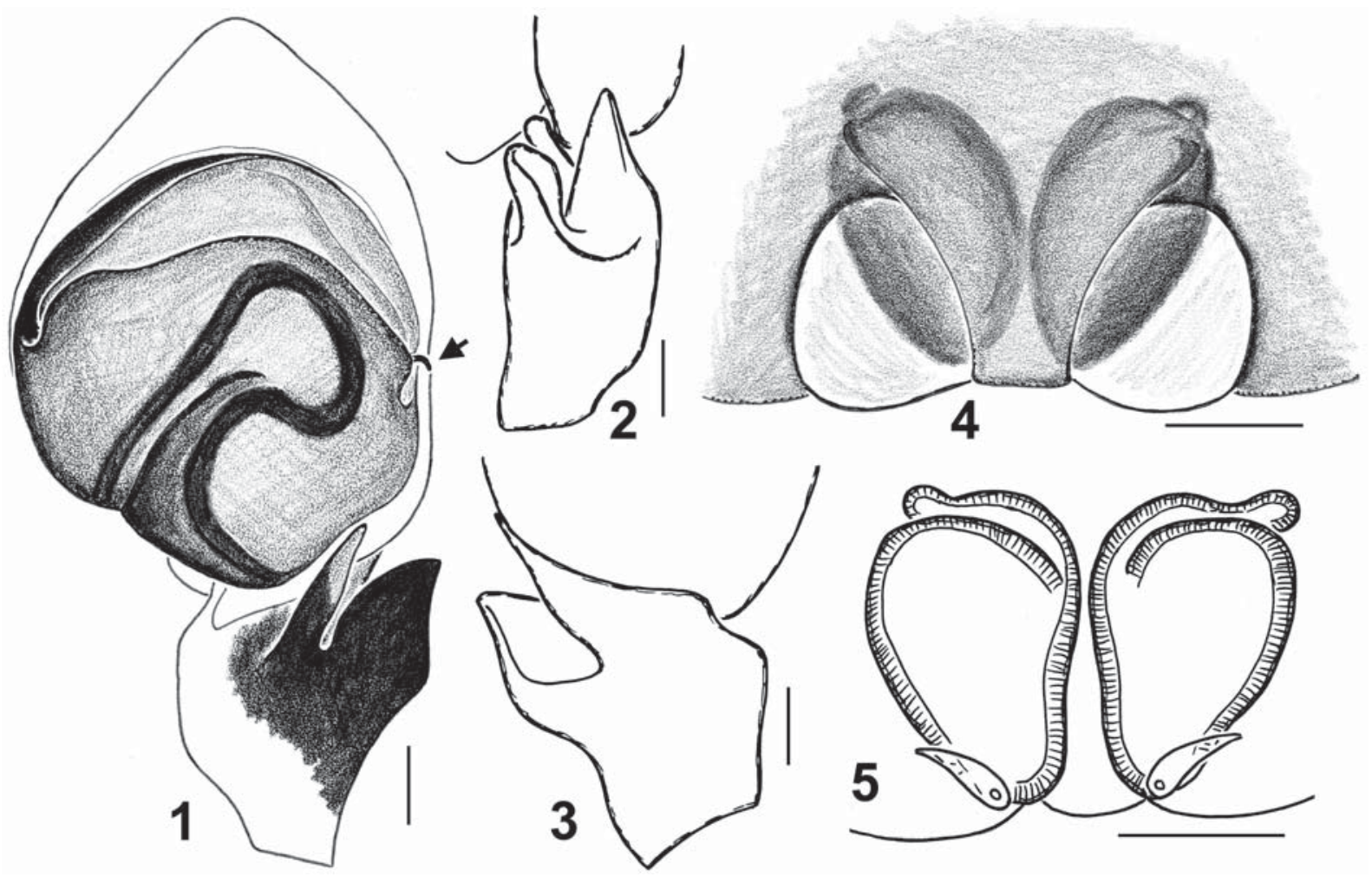

Figs 1-5: Artanes bucaensis sp.n. (1-3; $0^{\top}$ holotype) and Thanatus nitidus sp.n. (4-5; 9 paratype): 1 - male palp, ventral view; 2 tibial apophyses, lateral view; 3 - ditto, dorsal view; 4 - epigyne, ventral view; 5 - spermathecae, dorsal view. Scale lines: $0.1 \mathrm{~mm}$.

Рис. 1-5: Artanes bucaensis sp.n. (1-3; О голотип) and Thanatus nitidus sp.n. (4-5; + паратип): 1 - пальпа самца, вентрально; 2 - тибиальные отростки, латерально; 3 - тоже, дорзально; 4 - эпигина, снизу; 5 - сперматека, сверху. Масштаб: 0,1 мм.

$1.88+1.00$ (8.56). Leg spination of leg I: Fm d 0-1-0, pr 0-1-1-0; Tb v 2-2; Mt v 2-2-0. Colouration. Carapace light whitish yellow, with two longitudinal brownish bands and central elongate spot in the area of fovea. Sternum whitish, with brown specks and hairs. Labium, maxillae and chelicerae yellow. Abdomen light yellow, dorsum with a typical Thanatus-kind colour pattern: dark brown cardial spot and two lateral brownish stripes; venter yellow. Book-lung covers and spinnerets whitish. All legs and palps whitish with numerous brown specks, covered with protruding brown hairs. Epigyne and spermathecae as in Figs 4-5.

Thanatus oblongiusculus (Lucas, 1846) Map 3.

Identification: Logunov [1996: 167, figs 118-124, sub. T. constellatus].

MATERIAL. 1 ㅇ (MTAS), Taşburun Vil. [38 $9^{\circ} 43.96^{\prime \prime} \mathrm{N}$ $\left.37^{\circ} 12^{\prime} 18.58^{\prime \prime E}\right]$, Elbistan Distr., Kahramanmaraş Prov., 17.06.2006, EAY.

DISTRIBUTION. A European-Central Asian subboreal species, occurring from Spain [Urones, 1995: sub. Paratibellus o.] and France [Simon, 1932: sub. Paratibellus o.], eastward to NW China (Xinjiang) [Logunov, 1996: sub. T. constellatus] and southward to Tadjikistan [Lyakhov, 2000: sub. T. constellatus].

\section{Thanatus pictus L. Koch, 1881 \\ Map 3.}

Identification: Logunov [1996: 139, figs 10-14, sub Apollophanes babaly]; Szita \& Samu [2000: 168, figs 28-30, 35-36].

MATERIAL. Turkey: 5 qo (MTAS), Gölbaş1 Distr. [37³6'10.85"N, 37³9'12.73"E], Adiyaman Prov., 11.03.2007, KBK; 16 우우 (MTAS), Sarısalkım Vil. [37²'24.57"N, 37¹6'43.19"E], Gaziantep Prov., 8-26.04.2004, EAY; 1 + (MTAS), same locality, 29.06.2004, EAY; 2 우 (MTAS), Gaziantep Prov.

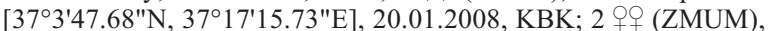
c. $1 \mathrm{~km}$ N of Parlak Vil. [38 36'0.96"N, 26 23'15.24"E], Karaburun Distr., İzmir Prov., 27.12.2009, KBK; 19 (MTAS), Bergama Distr. [39 $7^{\circ} 21.97^{\prime \prime N}, 27^{\circ} 11^{\prime} 28.43^{\prime \prime E}$, İzmir Prov., 01.05.2009,

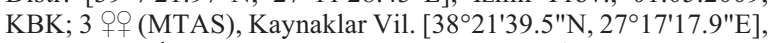
Buca Distr., İzmir Prov., 04.04.2009, KBK; 1 우 (MTAS), crossroad c. $2 \mathrm{~km} \mathrm{~S}$ of Emiralem [38 $37^{\prime} 0.48^{\prime \prime} \mathrm{N}, 27^{\circ} 6^{\prime} 48.80^{\prime \prime E}$ ], Menemen Distr., İzmir Prov., 19.04.2009, KBK; 1 q (MTAS), Yalıkavak Town [37 $7^{\prime} 13.31^{\prime \prime N}, 27^{\circ} 16^{\prime} 22.07^{\prime \prime E}$, Bodrum Distr., Küdür Peninsula, Muğla Prov., 23.04.2009, KBK; 1 ऽ (MTAS), Honaz Distr. [3745'19.48"N, 29¹5'14.67"E], Denizli Prov., 17.10.2009, KBK; 1 ㅇ (MTAS), Kurtboğazı Dam [40²1'14.09"N, 3240'51.39"E], on the road between Kizilcahamam and Ankara, Ankara Prov., 27.V.2009, KBK; 1 ㅇ (MTAS), Belören Vil. [37³8'12.13"N, 38³5'23.68"E], Kahta Distr., Adıyaman Prov., 05.04.2008, KBK; 1 ( (MTAS), crossroad nr. Ozanlı [36 58'37.93"N, 37¹0'18.10"E], Sahinbey Distr., Gaziantep Prov., 17.03.2007, KBK; 1 ㅇ (MTAS), c. $4 \mathrm{~km} \mathrm{~S}$ of Pazarc1k [37 $27^{\prime} 24.12^{\prime N}$, 37 $\left.7^{\circ} 4^{\prime} 40.05^{\prime \prime E}\right]$, Pazarc1k

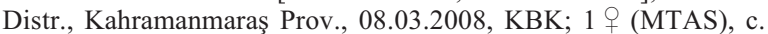
$3.5 \mathrm{~km}$ SW of Kizılenis Vil. [37²0'41.25"N, 3646'47.44"E], Türkoğlu Distr., Kahramanmaraş Prov., 09.03.2008, KBK; 1 + (MTAS), 


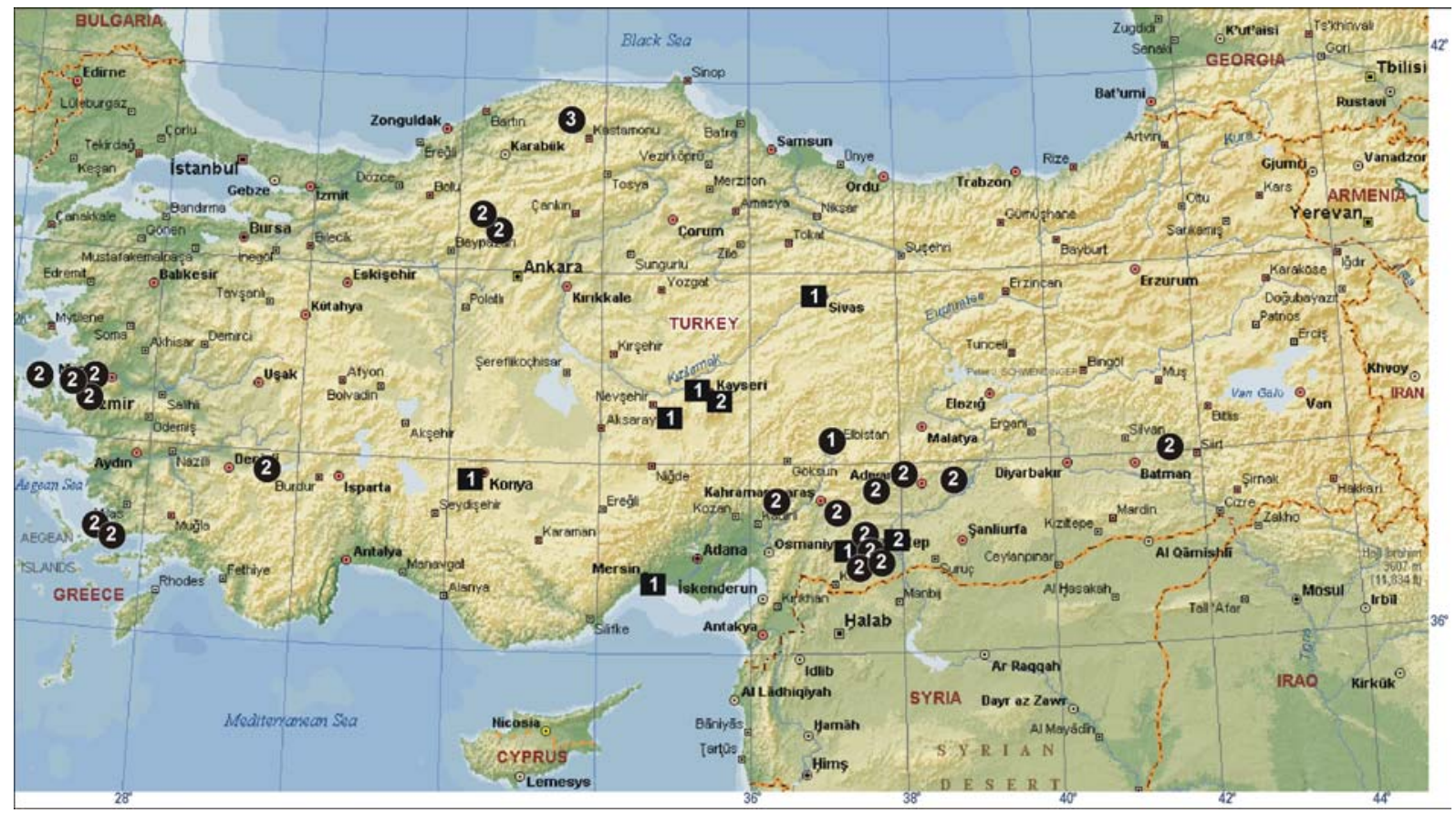

Map 3. Collection localities of Philodromidae in Turkey: Thanatus oblongiusculus (1), T. pictus (2) and T. sabulosus (3). Circles original data, squares - literature-derived data [Demir, 2008; Logunov \& Huseynov, 2008].

Карта 3. Находки Philodromidae в Турции: Thanatus oblongiusculus (1), T. pictus (2) и T. sabulosus (3). Кружки - оригинальные данные, квадраты — литературные данные [Demir, 2008; Logunov \& Huseynov, 2008].

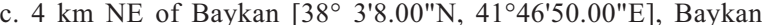
Distr., Siirt Prov., 18.05.2009, KBK; 1 ㅇ (MTAS), crossroads nr. Camlidere Town $\left[40^{\circ} 32^{\prime} 42.54 " \mathrm{~N}, 32^{\circ} 30^{\prime} 0.00^{\prime \prime} \mathrm{E}\right]$, Kizılcahamam Distr., Ankara Prov., 28.V.2009; KBK; 2 우 (MTAS), Burc Forest $\left[37^{\circ} 1^{\prime} 48.20^{\prime \prime N}, 37^{\circ} 17^{\prime} 56.65^{\prime \prime E}\right]$, Sahinbey Distr., Gaziantep Prov., 22.04.2007, KBK; 2 of (ZMUM), Aspat (Akyarlar) Bay [3659'28.74"N, 27¹9'20.85"E], Bodrum Distr., Muğla Prov., 24.12.2009, KBK; 1 † (MTAS), Meryem Uşağ 1 Vil., [3748'6.68"N, 3751'41.83"E] Tut Distr., Adiyaman Prov., 03.06.2007, KBK

DISTRIBUTION. A Euro-West Siberian sub-boreal species, known from central Europe [Szita \& Samu, 2000; Canard, 2005] to the Altai in east [Logunov, 1996: sub Apollophanes babaly].

Thanatus sabulosus (Menge, 1875) Map 3.

Identification: Logunov [1996: 177, figs 143-145, 153-157]; Szita \& Samu [2000: 163, figs 10-12, 18-19].

MATERIAL. TuRKey: $10^{7}$ (ZMUM), Azdavay Distr. [41 $\left.{ }^{\circ} 41.938^{\prime} \mathrm{N}, 33^{\circ} 25.971^{\prime} \mathrm{E}\right]$, Kastamonu Prov., $975 \mathrm{~m}$ a.s.l., 30.05.2009, YMM.

DISTRIBUTION. A Euro-East Siberian sub-boreal species, known from Spain [Urones, 1995] to Transbaikalia [Logunov, 1996]. This is a new record to the Turkish spider fauna.

\section{Thanatus vulgaris Simon, 1870}

Map 4.

Identification: Logunov [1996: 196, figs 194-197, 204-206]; Szita \& Samu [2000: 173, figs 40-42, 45-46].
MATERIAL. Turkey: 2 OO (MTAS), Aspat Bay [36 59'28.74"N, 27ํ'19'20.85"E], Bodrum Distr., Muğla Prov., 28.02.2009, KBK; $10^{7} 1$ ㅇ (MTAS), Kuyucak Vil. [3740'16.90"N, 38 16'56.60"E], Adıyaman Prov., 09.06.2007, KBK; $10^{7}$ (MTAS), forest located in the site of Demirçelik [36 $44^{\prime} 5.51^{\prime \prime N}, 36^{\circ} 13^{\prime} 6.88^{\prime \prime} \mathrm{E}$ ], İskenderun Distr., Hatay Prov., 18.05.2008, KBK; 1 ( (MTAS), Belen Pass [36 $\left.28^{\prime} 54.04^{\prime \prime} \mathrm{N}, 36^{\circ} 15^{\prime} 41.14^{\prime \prime} \mathrm{E}\right]$, Belen Distr., Hatay Prov., 10.05.2008, KBK; 1 क (MTAS), c. $1 \mathrm{~km} \mathrm{~N}$ of Parlak Vil. [38 36'0.96"N, 26²3'15.24"E], Karaburun Distr., İzmir Prov., 06.06.2009, KBK; 1 + (MTAS), Çiçekalan Vil. [3652'56.01"N, 38²' $1.99^{\prime \prime E], ~ B i r e c i k ~ D i s t r ., ~ S ̧ a n l ı u r f a ~ P r o v ., ~ 05.05 .2008, ~ K B K ; ~}$

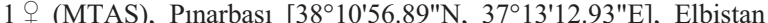
Distr., Kahramanmaraş Prov., 31.05.2008, KBK; 1 \& (MTAS), Çağlar Vil. [376'46.40"N, 4113'33.71"E], Nusaybin Distr., Mardin Prov., 13.05.2007, KBK; $10^{7} 1$ ㅇ (ZMUM), Kirıkhan Distr. [36³0'3.58"N, 36²0'46.37"E], Hatay Prov., 15.04.2007, KBK.

DISTRIBUTION. A Holarctic polyzonal species [Logunov, 1996; Lyakhov, 2000]; its occurrence in North America is known to be due to human introduction [Dondale \& Redner, 1978].

Tibellus Simon, 1875

The genus Tibellus contains some 53 species described worldwide [Platnick, 2010], many of which remain poorly studied. The Turkish fauna numbers 2 common species [Demir, 2008; present data].

\section{Tibellus macellus Simon 1875}

Map 4.

Identification: Maurer \& Walter [1984: 67, figs 1b,d]; Urones [1986: 240, figs 9a-e]. 


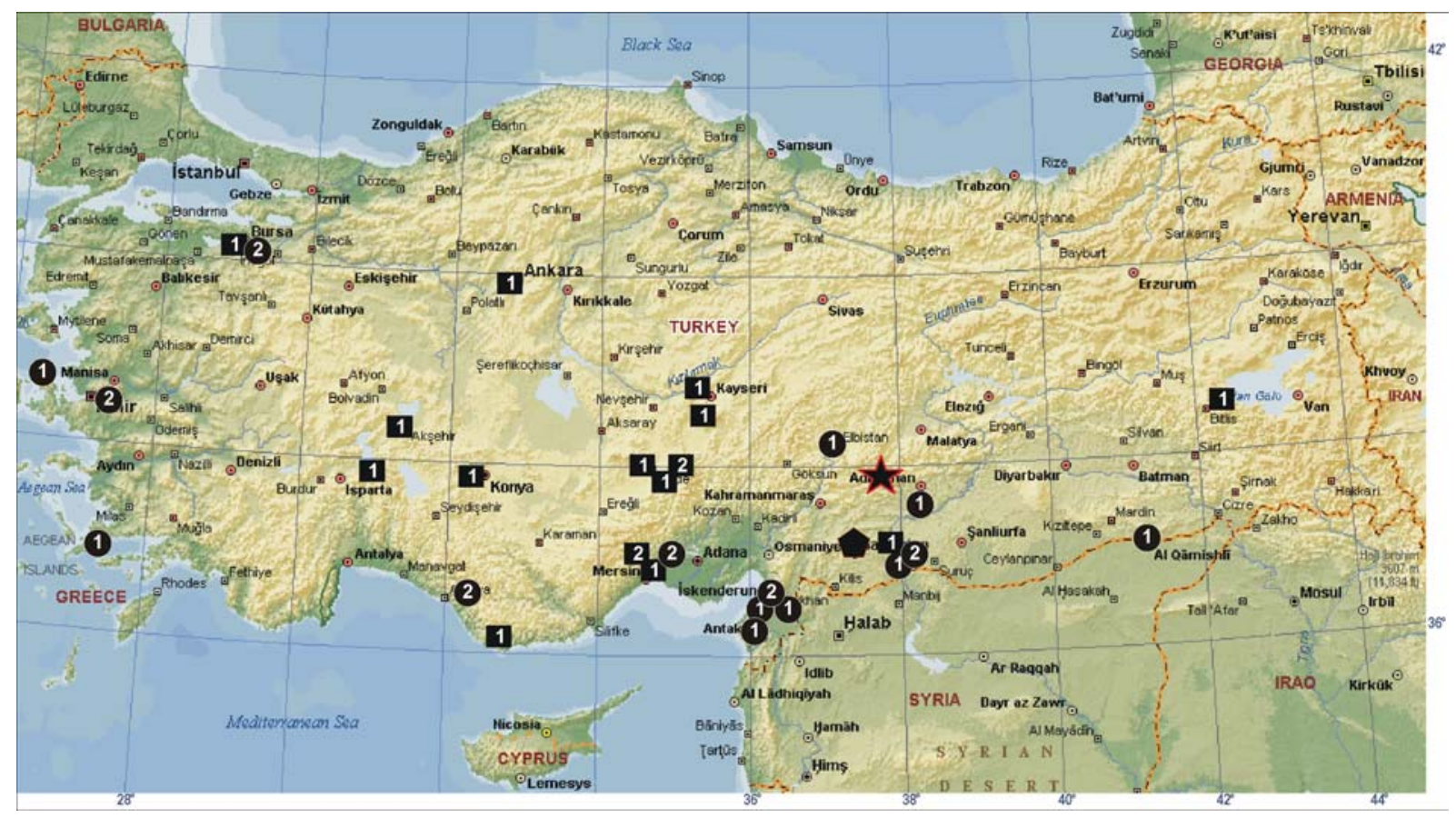

Map 4. Collection localities of Philodromidae in Turkey: Thanatus nitidus ( $\star$ - type locality, pentagon — locality of paratypes), $T$. vulgaris (1) and Tibellus macellus (2). Circles — original data, squares — literature-derived data [Demir et al., 2008; Demir, 2008; Logunov \& Huseynov, 2008].

Карта 4. Находки Philodromidae в Турции: Thanatus nitidus ( — типовое местонахождение, пятиугольник — местонахождение паратипов), T. vulgaris (1) и Tibellus macellus (2). Кружки — оригинальные данные, квадраты — литературные данные [Demir et al., 2008; Demir, 2008; Logunov \& Huseynov, 2008].

MATERIAL. TURKEY: 1 ( $($ MTAS), Dim Valley [36³2'3.33"N 32॰5'19.89"E], Kestel Town, Alanya Distr., Antalya Prov., 22.IV.2009, KBK; 7 Ơ $^{\top} 5$ 우 (ZMUM), campus of Uludağ Univ. [40 $\left.13.549^{\prime} \mathrm{N}, 28^{\circ} 52.109^{\prime} \mathrm{E}\right]$, Gorukle Vil., Bursa Prov., $423 \mathrm{~m}$ a.s.1., 2-3.06.2009, YMM; $1 \mathrm{O}^{7} 1$ ㅇ (MTAS), Orhangazi Vil.

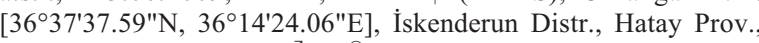
09.05.2008, KBK; $10^{7} 1$ 을 (MTAS), Yukarý Habip Vil. [37 $\left.5^{\prime} 45.62 " \mathrm{~N}, 37^{\circ} 54^{\prime} 48.54^{\prime \prime E}\right]$, Birecik Distr., Şanlıurfa Prov., 24.04.2009, KBK; 1 † (MTAS), forest nr. Demirçelik [36 $\left.46^{\circ} 5.51 " \mathrm{~N}, 36^{\circ} 13^{\prime} 6.88^{\prime \prime} \mathrm{E}\right]$, İskenderun Distr., Hatay Prov., 18.05.2008, KBK; $1 \mathrm{O}^{7}$ (MTAS), nr. Fetrek Cave [38 20'46.62"N, $\left.2^{\circ} 25^{\prime} 16.26^{\prime \prime E}\right]$, Vişnelik Vil., Kemalpaşa Distr., İzmir Prov., 05.VI.2009, KBK; $10^{7}$ (MTAS), Orhangazi Vil. [36 37'37.59"N, 36 14'24.06"E], İskenderun Distr., Hatay Prov., 09.05.2008, KBK; $10^{7}$ (LMNM), Kesbuku Vil. [36 55'55.13"N, 3451'34.58"E], on the road of Tarsus - Çamlýyayla Plateau, Mersin Prov., edge of field track on herb-rich hillside, 11.06.1993, CF \& SJ.

DISTRIBUTION. A Palaearctic sub-boreal species, known from south France and Spain in the west [Maurer \& Walter, 1984] to the Russian Far East [Efimik, 1999].

\section{Discussion}

To sum up, the currently known philodromid fauna of Turkey consists of 37 species [Bayram et al., 2008; Demir, 2008, Logunov \& Huseynov, 2008; Muster, 2009a; present data]. Although this number of species is higher than those of neighbouring countries, such as Greece (27 species [Bosmans \& Chatzaki, 2005]), Azerbaijan (22 species [Logunov \& Huseynov, 2008]) or
Israel (19 species [Levy, 1977, 1991, 1999]), it is hardly exhaustive. For instance, such species as Philodromus emarginatus (Schrank, 1803), P. medius O. Pickard-Cambridge, 1872, $P$. naxcivanicus Logunov et Huseynov, 2008, P. praedatus O. Pickard-Cambridge, 1871, known from the neighbouring regions of Greece or Azerbaijan [Bosmans \& Chatzaki, 2005; Logunov \& Huseynov, 2008] beyond doubt also occur in Turkey. To date, no representatives of the recently erected genus Halodromus Muster, 2009 has been collected from Turkey. This genus contains 6 valid species, of which three, viz. H. barbarae Muster, 2009; H. patellaris (Wunderlich, 1987) and H. pattelidens (Levy, 1977), occur in the Middle East [Levy, 1977; Muster, 2009b]; so, findings of Halodromus spp, at least in southern Turkey, are quite possible. A very conservative estimate of the entire diversity of Turkish philodromids could be at least 45 species.

ACKNOWLEDGEMENTS. We wish to express our warmest thanks to Mr G. Knight (of the LMNM), Dr Yu.M. Marusik (Magadan, Russia) and Dr. K.G. Mikhailov (of the ZMUM) for giving access to their collections. Mr Ersen Aydın Yağmur (İzmir; Turkey) is thanked for his valuable help during field studies. Dr Yu.M. Marusik kindly commented on the ms helping to improve it. Finally, Jon Cooter (Oxford, UK) is thanked for his kind help in editing the English of the final draft. 


\section{References}

Almquist S. 2006. Swedish Araneae, part 2, families Dictynidae to Salticidae // Insects Syst. Evol. Suppl. No.63. P.285-603.

Bayram A., Kunt K.B., Danışman T. 2008. Checklist of the Spiders of Turkey. Version 08.1.0. Online at: <http://www. spidersofturkey.com $>$.

Bosmans R., Chatzaki M. 2005. A catalogue of the spiders of Greece. A critical review of all spiders cited from Greece with their localities // Arachnological Contributions. Newsl. Belg. Arachnol. Soc. Vol.20. No.2, suppl. P.2-124.

Canard A. 2005. Catalogue of spider species from Europe and the Mediterranean basin. Parts I \& II // Rev. Arachnol. T.15. Fasc.3. P.1-255.

Demir H. 2008. An updated checklist of the Philodromidae (Araneae) of Turkey with zoogeographical remarks // Serket. Vol.11. No.1. P.7-12.

Demir H., Topçu A., Seyyar O. 2008. Contribution to the knowledge of the Philodromidae (Arachnida: Araneae) of Turkey // Zool. Middle East. Vol.43. P.118-120.

Dondale C.D., Redner J.H. 1975. The fuscomarginatus and histrio groups of the spider genus Philodromus in North America (Araneida: Thomisidae) // Can. Ent. Vol.107. P.369-384.

Dondale C.D., Redner J.H. 1978. The crab spiders of Canada and Alaska. Araneae: Philodromidae and Thomisidae // The Insects and Arachnida of Canada. Part 5. Canada. $255 \mathrm{pp}$

Gertsch W.J. 1933. New genera and species of North American spiders // Amer. Mus. Novit. No.636. P.1-28.

Koch C.L. 1837. Ubersicht des Arachnidensystems. Nurnberg. H.1 S.1-39.

Kubcová L. 2004. A new spider species from the group Philodromus aureolus (Araneae, Philodromidae) in central Europe // Thaler K. (ed.). Diversität und Biologie von Webspinnen, Skorpionen under anderen Spinnentieren. Denisia. Bd.12. S.291304.

Kulczyński W. 1903. Arachnoidea in Asia Minore et ad Constantinopolim a Dre F. Werner collecta // Sitz.-ber. Akad. Wiss. Wien. Bd.112. S.627-680.

Levy G. 1977. The philodromid spiders of Israel (Araneae: Philodromidae) // Israel J. Zool. Vol.26. P.193-229.

Levy G. 1991. On some new and uncommon spiders from Israel (Araneae) // Bull. Br. arachnol. Soc. Vol.8. Pt.7. P.227-232.

Levy G. 1999. New thomisid and philodromid spiders (Araneae) from southern Israel // Bull. Br. arachnol. Soc. Vol.11. Pt.5. P.185-190.

Logunov D.V. 1996. A critical review of the spider genera Apollophanes O. P.-Cambridge, 1898 and Thanatus C.L. Koch, 1837 in North Asia (Araneae, Philodromidae) // Rev. arachnol. T.11. Fasc.13. P.133-202.

Logunov D.V., Huseynov E.F. 2008. A faunistic review of the spider family Philodromidae (Aranei) of Azerbaijan // Arthropoda Selecta. Vol.17. No.1/2. P.117-131.

Lyakhov O.V. 2000 (for 1999). Contribution to the Middle Asian fauna of the spider genus Thanatus C. L. Koch, 1837 (Aranei: Philodromidae) // Arthropoda Selecta. Vol.8. No.4. P.221230

Marusik Yu.M. 1991. [Crab-spiders of the family Philodromidae (Aranei) of East Siberia] // Zool. Zhurnal. T.10. Vyp.10. S.4858 [in Russian with English summary].

Marusik Yu.M., Logunov D.V., Koponen S. 2000. Spiders of Tuva, South Siberia. Magadan: IBPS DVO RAS. 252 pp.
Maurer R., Walter J. E. 1984. Für die Schweiz neue und bemerkenswerte Spinnen (Araneae) II // Mitt. schweiz. ent. Ges. Bd.57. S.65-73.

Mcheidze T.S. 1997. [Spiders of Georgia: Systematics, Ecology, Zoogeographic Review]. Tbilisi Univ. 390 pp. [in Georgian].

Muster C. 2009a. Phylogenetic relationships within Philodromidae, with a taxonomic revision of Philodromus subgenus $\mathrm{Ar}$ tanes in the western Palearctic (Arachnida: Araneae) // Invertebr. Syst. Vol.23. P.135-169.

Muster C. 2009b. The Ebo-like running crab spiders in the Old World (Araneae, Philodromidae) // ZooKeys. Vol.16. P.47-73.

Muster C., Bosmans R, Thaler K. 2007. The Philodromus pulchellus-group in the Mediterranean: taxonomic revision, phylogenetic analysis and biogeography (Araneae: Philodromidae) // Invertebr. Syst. Vol.21. P.39-72.

Muster C., Thaler K. 2003. The Thanatus striatus species group in the eastern Alps, with description of Thanatus firmetorum sp. n. (Araneae: Philodromidae) // Bull. Br. arachnol. Soc. Vol.12. Pt.8. P.376-382.

Muster C., Thaler K. 2004. New species and records of Mediterranean Philodromidae (Arachnida, Araneae): I. Philodromus aureolus group // Thaler K. (ed.). Diversität und Biologie von Webspinnen, Skorpionen under anderen Spinnentieren. Denisia. Bd.12. S.305-326.

Ono H. 1988. A revisional study of the spider family Thomisidae (Arachnida, Araneae) of Japan. Tokyo: National Science Museum. $252 \mathrm{p}$.

Ono H., Ban M. 2009. Oxyopidae, Philodromidae // Ono H. (ed.). The Spiders of Japan with keys to the families and genera and illustrations of the species. Tokai Univ. Press, Kanagawa, pp. 249-250, 476-481.

Pavesi P. 1876. Gli aracnidi Turchi // Atti Soc. Ital. Sci. nat. Vol.19. No.1. P.1-27.

Platnick N. 2010. The World Spider Catalog, Version 10.5, (Philodromidae pages last updated October 30th, 2009), American Museum of Natural History. Online at: $<$ http://research.amnh. org/entomology/spiders/catalog/INTRO1.html>

Ponomarev A.V. 2007. [New spiders (Aranei) from the south-east of Europe] // Caucasian entomol. Bull. Vol.3. No.1. P.3-7 [in Russian with English summary].

Roberts M.J. 1995. Collins Field Guide: Spiders of Britain \& Northern Europe. London: HarperCollins. 383 pp.

Segers H. 1989. A redescription of Philodromus albidus Kulczyński, 1911 (Araneae, Philodromidae) // Bull. Br. arachnol. Soc. Vol.8. P.38-40.

Simon E. 1932. Les arachnides de France. Tome 6. Synopsis générale et catalogue des espèces françaises de l'ordre des Araneae; 4e partie. Paris. P.773-978.

Szita É., Samu F. 2000. Taxonomical review of Thanatus species (Philodromidae, Araneae) of Hungary // Acta Zool. Acad. Sci. Hung. Vol.46. P.155-179.

Thorell T. 1870. On European spiders // Nov. Act. reg. Soc. sci. Upsaline. Vol.7. No.3. P.109-242.

Urones C. 1986. La familia Philodromidae (Araneae) en el centrooeste de la Península Ibérica // Boln Asoc. esp. Entomologia. Vol.10. P.231-244

Urones C. 1995. Catálogo y atlas de las Arañas de la familia Philodromidae Thorell, 1870 de la Península Ibérica e Islas Baleares // Graellsia. Vol.51. P.55-81.

Wunderlich J. 1992 (for 1991). Die Spinnen-Fauna der Makaronesischen Inseln: Taxonomie, Ökologie, Biogeographie und Evolution // Beitr. Araneol. Bd.1. S.1-619. 